\title{
Usefulness of Liver and Spleen Acoustic Radiation Force Impulse (ARFI) for the Evaluation of Cirrhotic Patients
}

\author{
Luciana Barbosa ${ }^{1}$, Catarina Oliveira' ${ }^{1}$ Alexandra Fernandes ${ }^{2}$, Margarida Marques ${ }^{3}$, \\ João Pereira ${ }^{3}$, Carlos Sofia ${ }^{2}$, Filipe Caseiro-Alves', Maria José Noruegas' ${ }^{1}$ \\ ${ }^{1}$ Medical Imaging Department, Faculty of Medicine, University Hospital of Coimbra, Coimbra, Portugal \\ ${ }^{2}$ Gastroenterology Department, University Hospital of Coimbra, Coimbra, Portugal \\ ${ }^{3}$ Laboratory of Biostatistics and Medical Informatics, Institute for Biomedical Imaging and Life Sciences, Faculty \\ of Medicine, University of Coimbra, Coimbra, Portugal \\ Email: lucianabernardesbarbosa@gmail.com
}

Received 2 September 2014; revised 10 October 2014; accepted 11 November 2014

Copyright (C) 2014 by authors and OALib.

This work is licensed under the Creative Commons Attribution International License (CC BY).

http://creativecommons.org/licenses/by/4.0/

(c) (i) Open Access

\section{Abstract}

Objective: To evaluate the correlation between ARFI and Child-Pugh classification. Secondary Aims: 1) To compare ARFI values (hepatic, splenic and spleno-hepatic index) from cirrhotic to normal population; 2) To correlate biochemical parameters of liver function and ARFI. Materials and Methods: 58 cirrhotic patients (referenced to US for surveillance or to clarify any hepatic decompensation) were included in this prospective study, as well as $\mathbf{3 8}$ healthy subjects who underwent ultrasonography for other reasons than hepatic evaluation. All had ARFI liver and spleen evaluation on ACUSON S2000 ARFI equipment. The best cut-off liver and spleen values for the diagnosis of cirrhosis in comparison to the normal subjects were determined using SPSS ${ }^{\circledR} \mathrm{v20}$. Results: Mean liver ARFI values in controls and cirrhotic patients were respectively $1.18 \pm 0.22 \mathrm{~m} / \mathrm{s}$ and $2.93 \pm 0.50 \mathrm{~m} / \mathrm{s}$. The ROC curve demonstrated an AUC 0.998 and the best cut-off was 1.89 . Mean spleen ARFI values in controls and cirrhotic patients were respectively $2.60 \pm 0.42 \mathrm{~m} / \mathrm{s}$ and $3.03 \pm 0.71$. The ROC curve demonstrated an AUC 0.766 and the best cut-off was $2.73 \mathrm{~m} / \mathrm{s}$. The splenohepatic index showed a worse AUC than ARFI liver. A weak correlation was found between the ARFI liver and Child-Pugh. We found no statistically significant differences for spleen ARFI values and Child-Pugh. We found a statistically significant correlation between liver ARFI and bilirubin, ALKP, GGT, AST and AST/ALT ratio; and with spleen ARFI and ALKP and AST/ALT ratio. Conclusion: We showed that there is a tendency of higher levels of liver ARFI values for higher Child-Pugh classification suggesting a definite trend for higher values with more severe disease.

\section{Keywords}

Liver Cirrhosis, Child-Pugh Classification, Liver Stiffness, Spleen Stiffness, ARFI

How to cite this paper: Barbosa, L., Oliveira, C., Fernandes, A., Marques, M., Pereira, J., Sofia, C., Caseiro-Alves, F. and Noruegas, M.J. (2014) Usefulness of Liver and Spleen Acoustic Radiation Force Impulse (ARFI) for the Evaluation of Cirrhotic Patients. Open Access Library Journal, 1: e916. http://dx.doi.org/10.4236/oalib.1100916 


\section{Introduction}

Cirrhosis is a commonly encountered chronic liver disease characterized by the deposition of fibrous tissue within the liver. This causes the liver to become stiffer than normal, with distortion of normal liver architecture [1].

A precise estimation of the degree of liver fibrosis is important for the estimation of prognosis, surveillance, and treatment decision in patients with chronic liver disease [2] [3].

Biopsy provides an extremely valuable contribution to the assessment of liver status in the case of chronic disease, offering information both on fibrosis and necro-inflammatory activity. However, not only the risk of complications, which has been reported with a frequency of $5 \%-20 \%$ for minor complications and $0.3 \%-0.5 \%$ for major complications [4] including exceptional cases of mortality, but also contraindications, such as coagulopathy, large volume ascitis, poor patient cooperation and lack of patient consent, tend to limit its use, especially for repeated procedures over time [5].

Acoustic Radiation Force Impulse (ARFI) imaging Virtual Touch ${ }^{\mathrm{TM}}$ tissue quantification uses a software of the US Scanners Siemens Acuson S2000 (Siemens Healthcare, Erlangen, Germany) that offers the possibility of performing a quantitative measurement of the elasticity of the hepatic parenchyma during conventional US evaluations [6]. ARFI imaging technology involves the mechanical excitation of tissue using short-duration acoustic pulses in a region of interest chosen by the examiner, producing shear waves that spread away from the region of interest, generating localized, micron-scale displacements in the tissue [7] [8]. By recording the shear wave front at several locations and correlating these measurements with the elapsed time, the shear wave velocity $(\mathrm{m} / \mathrm{s})$ can be quantified; generally, the stiffer is a region in the tissue; the shear wave velocity will be greater as it travels through this region [9]-[12].

The Child-Pugh Score stratifies the severity of cirrhosis (from class A-better prognosis to class C-worst prognosis), and predicts the probability of decompensation and death, using clinical and analytical data (ascitis, encephalopathy, total bilirubin, serum albumin and international normalized ratio or prothrombin time). Originally, the Child-Pugh Score was used to predict mortality during surgery; however it is now used to assess prognosis and to evaluate the need of liver transplantation.

The primary aim of this study was to evaluate the correlation between ARFI results and Child-Pugh classification. Secondary aims were 1) to compare ARFI values (hepatic, splenic and spleno-hepatic index) from cirrhotic to normal population and 2) to correlate biochemical parameters of liver function and ARFI.

\section{Methods and Materials}

\subsection{Subjects}

We obtained written informed consent from all the subjects in the study and the approval of the Hospital Ethics Committee.

In this prospective study 96 examinees were categorized into two groups: 1) 58 patients with previously diagnosed liver cirrhosis (biopsy-proven cirrhosis or clinically presumed cirrhosis-combination of clinical, analytical, endoscopic and imaging data); 2) 38 healthy individuals, with no hepatic disease, who undergone ultrasonography study for various reasons-abdominal pain, vomiting, renal colic and some volunteer members of hospital staff (the inclusion criteria were no history of liver disease, no diseases such as diabetes, renal insufficiency, and congestive heart failure, and laboratory tests done within 30 days. The exclusion criteria were age under 18 years old, use of chronic medication and refusal to participate in the study);

Diagnosis of cirrhosis by non-invasive imaging, including ultrasound, magnetic resonance or a combination of the two, was established based on typical imaging findings, including liver surface nodularity, liver segment I hypertrophy, splenomegaly, hepatofugal portal venous flow and portosystemic vascular shunts. The exclusion criteria were: no laboratory tests done within 30 days and portal vein thrombosis.

The 58 cirrhotic patients were categorized using the Child-Pugh classification: Child A (34, 58.6\%), Child B (13, 22.4\%), Child C (10, 19\%). 
Etiology of liver cirrhosis included alcohol ( $n=47,81 \%)$, hepatitis B virus $(\mathrm{HBV})$ infection $(\mathrm{n}=4,6.9 \%)$, hepatitis $C$ virus $(\mathrm{HCV})$ infection $(n=4,6.9 \%)$ and primary biliary cirrhosis $(\mathrm{PBC})(\mathrm{n}=3,5.2 \%)$.

Demographic data, including age and gender were recorded for each individual patient at the time of ARFI imaging.

\subsection{Acoustic Radiation Force Impulse Imaging Examination}

All participants $(\mathrm{n}=96)$ were examined using the Siemens ACUSON S2000 Ultrasound ARFI Virtual Touch ${ }^{\mathrm{TM}}$ Tissue Quantification System (Erlangen, Germany). During real-time B-mode imaging, a region-of-interest was placed in right hepatic lobe or the spleen on vessel- and tumor-free parenchyma, at a depth of $2-8 \mathrm{~cm}$, during suspended respiration (Figure 1(a) and Figure 1(b)). In 6 hours fasting examinees, six measurements were performed from both referenced organs. The final ARFI value for each participant and for each organ was calculated as a mean of these 6 measurements. We used a $4 \mathrm{MHz}$ convex probe that applies short duration acoustic pulses with a fixed transmitter frequency to generate localized displacements in tissue. The results are expressed in meters per second $(\mathrm{m} / \mathrm{s})$.

ARFI values in $\mathrm{m} / \mathrm{s}$ obtained in the liver were multiplied by those of the spleen and divided by 100 , producing the spleno-hepatic index.

\subsection{Biochemical Parameters of Liver Function}

The following biological parameters were recorded in each individual patient at the same month of ARFI examination: total bilirubin (Bilirubin) ( $\mu \mathrm{mol} / \mathrm{L}$ ), alkaline phosphatase (ALKP) (U/L), $\gamma$-glutamyl transpeptidase (GGT) (U/L), aspartate aminotransferase (AST) (U/L) and alanine aminotransferase (ALT) (U/L). We calculated also the AST/ALT ratio.

\subsection{Statistical Analysis}

Statistical analysis was performed using the Statistical Package for Social Sciences software (IBM SPSS Statistics for Windows, Version 20.0. Armonk, NY: IBM Corp.). The verification of the assumptions of normality for the application of parametric tests on the variables of interest was done resorting to the Kolmogorov-Smirnov test with Lilliefors correction. Non-parametric tests were performed whenever normality could not be assumed. All tests were two-tailed and a p-value of less than 0.05 was deemed to be statistically significant.

For ARFI imaging, the mean of 6 successful measurements obtained in each patient for liver and for spleen was calculated and used for further analyses. Overall comparison of ARFI liver and spleen values between different Child-Pugh classification groups performed using the Mann-Whitney U-test. These results were expressed as mean \pm standard deviation for the sake of comparability with published data. Laboratory tests were compared using the Mann-Whitney U-test and the results were expressed as median (range).

For the purpose of evaluating the performance of hepatic fibrosis tests in predicting cirrhosis, using clinical assessment as the gold standard, ROC analyses were performed. This included the calculation of the area under the ROC curves (AUC), as well as related sensitivity (Se) and specificity (Sp) values.

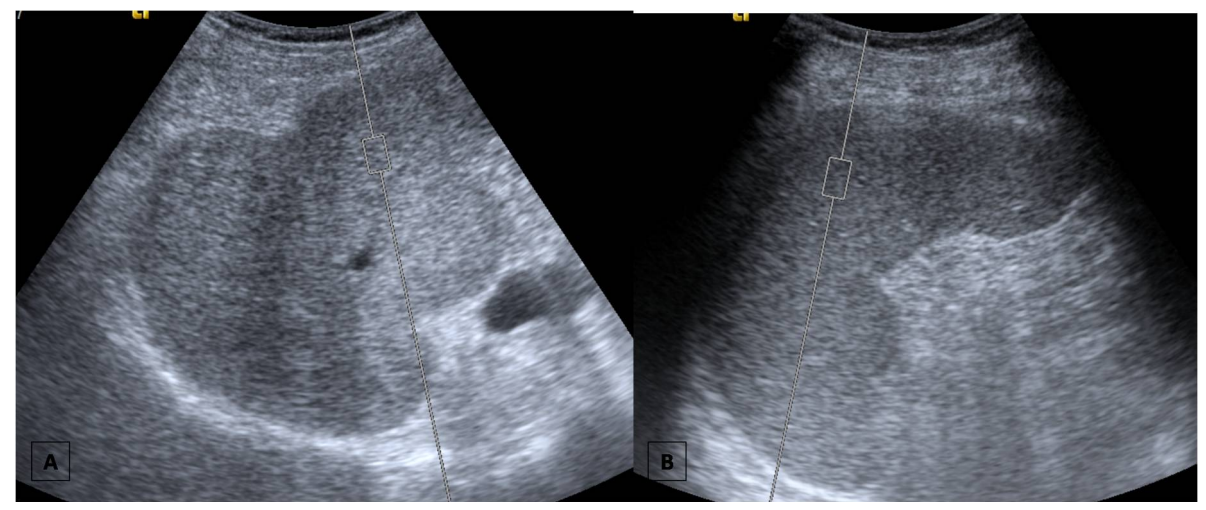

IFigure 1. ARFI measurements in a cirrhotic patient. (A) Right liver lobe; (B) Spleen. 
The Spearman correlation between Child-Pugh classification and ARFI measurements for liver and spleen was calculated only for cirrhotic patients.

The chi-square test was used to compare categorical variables, expressed as percentages. Logistic regression analysis was used to confirm predictors of cirrhosis while removing the nuisance influence of sex and age.

\section{Results}

\subsection{Patient Population Characteristics}

In total 96 consecutive patients were evaluated by ARFI. ARFI was able to provide valid results in all of these patients. The etiology of liver disease (alcohol, HBV, HCV and PBC) and other patient characteristics are listed in Table 1.

58 cirrhotic patients (A) and 38 healthy individuals (B), were included in the study. Mean age for group A was $65.5 \pm 11.8$ and for group B was $56.7 \pm 16.3$. As expected, the patients with liver cirrhosis were older compared to the other group. In the group A $73.9 \%$ were male and $19.4 \%$ were female, while in group B $34.2 \%$ were male and $65.8 \%$ were female.

There was a significant difference for age and gender between the two groups; however, applying a logistic regression, we found that these differences do not interfere in the predictive capability of ARFI liver and spleen values.

We found statistically differences between the serum markers results of the two groups (healthy and cirrhotic), with exception for ALT (Table 2).

\subsection{ARFI Liver Values}

Six ARFI values were successfully obtained from the right lobe of the liver in all 96 patients.

There was a significant difference in the ARFI hepatic values between the two groups. The mean ARFI hepatic values of cirrhotic and healthy groups were $2.93 \pm 0.50 \mathrm{~m} / \mathrm{s}$ and $1.18 \pm 0.22 \mathrm{~m} / \mathrm{s}$, respectively $(\mathrm{p}<0.001)$ (Figure 2 and Table 3). The ROC curve analysis demonstrated an AUC of 0.998 (95\% Confidence Interval $0.994-1.000$ ). The best cut-off for establishing the presence of cirrhosis proved to be $1.89 \mathrm{~m} / \mathrm{s}$ for liver values (98.3\% Se and $100 \% \mathrm{Sp}$ ).

\subsection{ARFI Spleen Values}

Six ARFI values were successfully obtained from the spleen of all 96 patients, regardless of splenic size (whether normal or enlarged). The mean ARFI spleen values were $2.60 \pm 0.42 \mathrm{~m} / \mathrm{s}$ in normal subjects and $3.03 \pm 0.71$

Table 1. Patients characteristics, values are expressed as mean \pm SD or $\mathrm{n}(\%)$.

\begin{tabular}{|c|c|c|c|}
\hline & Cirrhosis (A) & Healthy (B) & $\mathrm{p}$ \\
\hline $\mathrm{N}$ & 58 & 38 & \\
\hline Age (yrs) & $65.5 \pm 11.8$ & $56.7 \pm 16.3$ & $\mathrm{p}=0.003^{*}$ \\
\hline Gender (M:F) & $\begin{array}{c}\text { M: } 73.9 \% ; \mathrm{F}: 19.4 \% \\
51: 7\end{array}$ & $\begin{array}{c}\text { M: } 34.2 \% ; \mathrm{F}: 65.8 \% \\
13: 25\end{array}$ & $\mathrm{p}<0.001^{* *}$ \\
\hline \multicolumn{4}{|c|}{ Etiology of liver disease } \\
\hline Alcohol & 47 (81\%) & & \\
\hline $\mathrm{HBV}$ & $4(6.9 \%)$ & & \\
\hline $\mathrm{HCV}$ & 4 (6.9\%) & & \\
\hline $\mathrm{PBC}$ & $3(5.2 \%)$ & & \\
\hline \multicolumn{4}{|c|}{ Child-Pugh classification } \\
\hline A & $34(58.6 \%)$ & & \\
\hline $\mathrm{B}$ & $13(22.4 \%)$ & & \\
\hline $\mathrm{C}$ & 10 (19\%) & & \\
\hline
\end{tabular}

*t-Test; **Test chi-square (F—female; HBV—hepatitis B virus; HCV—hepatitis C virus; M—male; PBC—primary biliary cirrhosis). 
Table 2. Laboratory tests results of healthy and cirrhotic patients. Results are express as median and min-max.

\begin{tabular}{cccc}
\hline & Cirrhosis $(\mathrm{A})$ & Healthy $(\mathrm{B})$ & $\mathrm{p}$ \\
\hline Bilirubin $(\mu \mathrm{mol} / \mathrm{L})$ & $20.5(7.0-4539)$ & $9.5(3.8-34.4)$ & $\mathrm{p}<0.001^{*}$ \\
ALKP (U/L) & $109(39-387)$ & $65.5(41-146)$ & $\mathrm{p}<0.001^{*}$ \\
GGT (U/L) & $92.5(13-647)$ & $22(10-163)$ & $\mathrm{p}<0.001^{*}$ \\
AST (U/L) & $41(18-273)$ & $27(13-56)$ & $\mathrm{p}<0.001^{*}$ \\
ALT (U/L) & $35(10-220)$ & $30(12-63)$ & $\mathrm{p}=0.259^{*}$ \\
AST/ALT & $1.1(0.32-4.88)$ & $0.9(0.39-1.44)$ & $\mathrm{p}=0.001^{*}$ \\
\hline
\end{tabular}

*Mann Whitney test (ALKP—alkaline phosphatase; ALT—alanine aminotransferase; AST—aspartate aminotransferase; Bilirubin—-total bilirubin; GGT- $\gamma$-glutamyl transpeptidase).

Table 3. Liver and splenic stiffness values in healthy and cirrhotic patients. Values are expressed as mean \pm SD.

\begin{tabular}{lccc}
\hline & Cirrhosis $(\mathrm{A})$ & Healthy (B) & p \\
\hline ARFI Liver $(\mathrm{m} / \mathrm{s})$ & $2.93 \pm 0.50$ & $1.18 \pm 0.22$ & $\mathrm{p}<0.001^{*}$ \\
ARFI Spleen $(\mathrm{m} / \mathrm{s})$ & $3.03 \pm 0.71$ & $2.60 \pm 0.42$ & $\mathrm{p}<0.001^{*}$ \\
\hline
\end{tabular}

*Mann Whitney test.

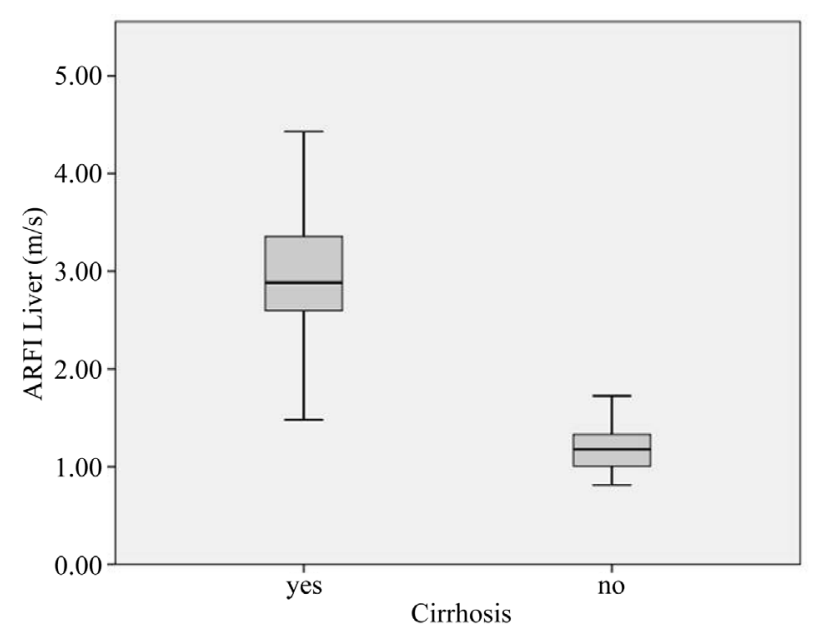

Figure 2. Box plots of liver ARFI values obtained in healthy and cirrhotic patients. A significant difference between healthy and cirrhotic was observed $(\mathrm{p}<0.001)$.

in cirrhotic patients.

We observed a statistically significant difference between ARFI spleen values in subjects without hepatic pathology and those with liver cirrhosis ( $<<0.001$ ) (Figure 3 and Table 3 ). The ROC curve analysis demonstrated an AUC of 0.766 (95\% Confidence Interval 0.671 - 0.862). The best cut-off for establishing the presence of cirrhosis proved to be $2.73 \mathrm{~m} / \mathrm{s}$ for spleen values (78.9\% Se and $65.8 \% \mathrm{Sp}$ ).

\subsection{Spleno-Hepatic Index}

We calculated the spleno-hepatic index (liver ARFI values multiplied by splenic values divided by 100) and concluded that the AUC for this index is subtle worse than AUC ARFI liver (data not shown).

\subsection{Correlation between ARFI and Child-Pugh Classification}

We studied 34 patients in Child-Pugh A group, 13 in Child-Pugh B and 10 in Child-Pugh C (Table 1). The mean ARFI liver values were $2.83 \pm 0.54 \mathrm{~m} / \mathrm{s}, 2.95 \pm 0.40$ and $3.24 \pm 0.46$, respectively. A weak Spearman correlation was found between the ARFI liver values and the Child-Pugh classification $(\rho=0.280 ; \mathrm{p}=0.039)$ (Figure 4). 


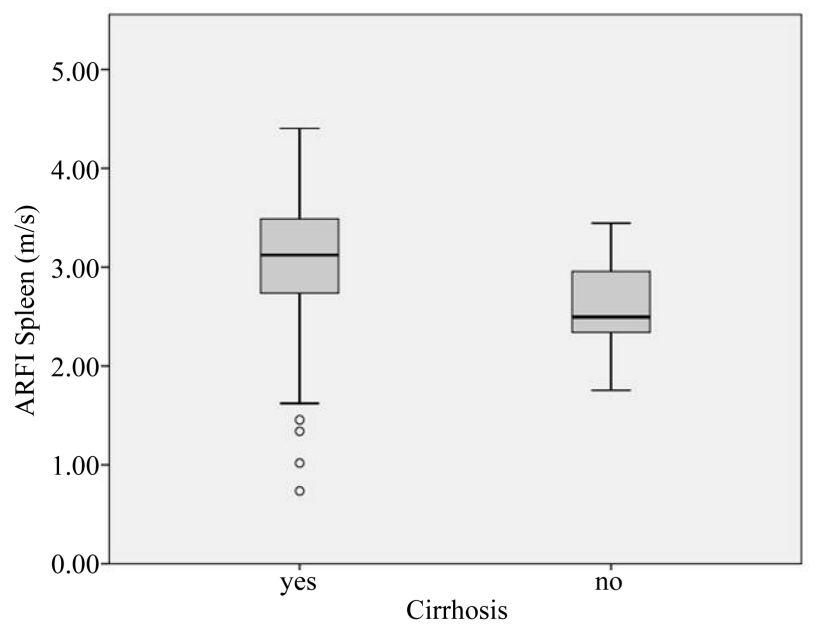

Figure 3. Box plots of spleen ARFI values obtained in healthy and cirrhotic patients. A significant difference between healthy and cirrhotic was observed $(\mathrm{p}<0.001)$.

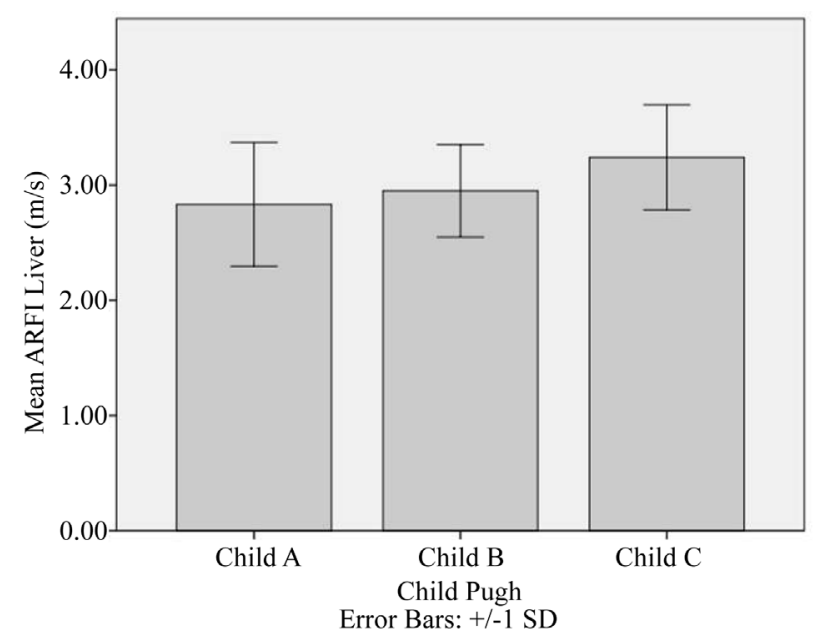

Figure 4. Column chart of liver ARFI mean values obtained in cirrhotic patients, classified in A, B and C of Child-Pugh classification. Error bars represent 1 standard deviation. A weak linear correlation was found between the ARFI liver values and the Child-Pugh classification.

On a closer inspection, there were no statistically significant correlation between spleen ARFI values and the Child-Pugh classification.

\subsection{Correlation between ARFI and Biochemical Parameters of Liver Function}

We examined the correlation between the ARFI liver and spleen values and results from laboratory tests (Table 4). We found that there is a statistically significant direct correlation between liver ARFI values and the laboratory results of bilirubin, ALKP, GGT, AST and AST/ALT ratio. In what concerns to spleen, we found a statistically significant direct correlation between the ARFI values and ALKP and AST/ALT ratio.

\section{Discussion}

Our results were consistent with those found on recent literature (Table 5).

Yu et al. [1] and Piscaglia et al. [5] compared ARFI liver values in normal and cirrhotic patients. Yu deter- 
Table 4. Correlation between ARFI results and laboratory tests results.

\begin{tabular}{llc}
\hline & ARFI liver & ARFI spleen \\
\hline \multirow{2}{*}{ Bilirubin } & $\boldsymbol{\rho}=\mathbf{0 . 5 2 8}$ & $\rho=0.210$ \\
ALKP & $\mathbf{p}<\mathbf{0 . 0 0 1}$ & $\mathrm{p}=0.060$ \\
& $\boldsymbol{\rho}=\mathbf{0 . 5 2 9}$ & $\boldsymbol{\rho}=\mathbf{0 . 3 6 9}$ \\
GGT & $\mathbf{p}<\mathbf{0 . 0 0 1}$ & $\mathbf{p}=\mathbf{0 . 0 0 1}$ \\
& $\boldsymbol{\rho}=\mathbf{0 . 4 7 9}$ & $\rho=0.160$ \\
AST & $\mathbf{p}<\mathbf{0 . 0 0 1}$ & $\mathrm{p}=0.171$ \\
& $\boldsymbol{\rho}=\mathbf{0 . 3 9 5}$ & $\rho=0.168$ \\
ALT & $\mathbf{p}<\mathbf{0 . 0 0 1}$ & $\mathrm{p}=0.129$ \\
& $\rho=0.084$ & $\rho=0.003$ \\
AST/ALT & $\mathrm{p}=0.455$ & $\mathrm{p}=0.980$ \\
& $\boldsymbol{\rho}=\mathbf{0 . 3 6 7}$ & $\boldsymbol{\rho}=\mathbf{0 . 2 5 0}$ \\
& $\mathbf{p}<\mathbf{0 . 0 0 1}$ & $\mathbf{p}<\mathbf{0 . 0 5}$ \\
\hline
\end{tabular}

$\rho$ - Spearman correlation coefficient; ALKP—alkaline phosphatase; ALT—alanine aminotransferase; AST—aspartate aminotransferase; Bilirubin—-total bilirubin; GGT— $\gamma$-glutamyl transpeptidase.

Table 5. Comparison between our results and previous studies results.

\begin{tabular}{|c|c|c|c|c|c|}
\hline & & \multicolumn{2}{|c|}{ ARFI Liver (m/s) } & \multicolumn{2}{|c|}{ ARFI Spleen (m/s) } \\
\hline & & Normal & Cirrhotic & Normal & Cirrhotic \\
\hline A. Gallotti [13] & 2010 & 1.59 & - & 2.44 & - \\
\hline & & & & $2.04 \pm 0.28$ & $3.10 \pm 0.55$ \\
\hline Simona Bota [14] & 2010 & - & - & \multicolumn{2}{|c|}{$\begin{array}{l}\text { AUC 0.91, cut-off } 2.51 \\
\text { (Se 85.2\%; Sp 91.7\%) }\end{array}$} \\
\hline & & 1.13 & 2.55 & & \\
\hline F. Piscaglia [5] & 2011 & \multicolumn{2}{|c|}{$\begin{array}{l}\text { AUC 0.941, cut-off } 1.75 \\
\text { (Se 93\%; Sp 85.1\%) }\end{array}$} & - & - \\
\hline & & $1.13 \pm 0.13$ & $2.77 \pm 0.68$ & $2.27 \pm 0.35$ & $3.29 \pm 0.65$ \\
\hline I. Grgurevic [16] & 2011 & \multicolumn{2}{|c|}{-} & \multicolumn{2}{|c|}{$\begin{array}{l}\text { AUC 0.822, cut-off } 2.73 \\
\text { (Se } 90 \% \text {; Sp } 77.8 \% \text { ) }\end{array}$} \\
\hline K. Rifai [17] & 2011 & $1.09 \pm 0.18$ & $2.55 \pm 1.02$ & $2.86 \pm 0.58$ & $3.25 \pm 0.56$ \\
\hline & & $1.16 \pm 0.34$ & $2.35 \pm 0.70$ & & \\
\hline Hojun Yu [1] & 2012 & \multicolumn{2}{|c|}{$\begin{array}{l}\text { AUC 0.932, cut-off } 1.36 \\
\text { (Se } 88 \% \text {; Sp 90\%) }\end{array}$} & - & - \\
\hline Vermehren J. [20] & 2012 & - & $3.01 \pm 0.99$ & - & $3.64 \pm 0.88$ \\
\hline Yoshitaka Takuma [15] & 2013 & 1.21 & 2.58 & 2.16 & 3.36 \\
\hline & & $1.18 \pm 0.22$ & $2.93 \pm 0.50$ & $2.60 \pm 0.42$ & $3.03 \pm 0.71$ \\
\hline Our study & 2013 & \multicolumn{2}{|c|}{$\begin{array}{l}\text { AUC 0.998, cut-off } 1.89 \\
\text { (Se } 98.3 \% \text {; Sp 100\%) }\end{array}$} & \multicolumn{2}{|c|}{$\begin{array}{c}\text { AUC } 0.766 \text {, cut-off } 2.73 \\
\text { (Se } 78.9 \% \text {; Sp } 65.8 \% \text { ) }\end{array}$} \\
\hline
\end{tabular}

AUC—area under curve; Se—sensitivity; Sp—specificity.

mine $1.13 \mathrm{~m} / \mathrm{s}$ for healthy and $2.55 \mathrm{~m} / \mathrm{s}$ for cirrhosis and Piscaglia determined 1.16 for healthy and $2.35 \mathrm{~m} / \mathrm{s}$ for cirrhotic patients. Our study found values slightly higher than those.

Gallotti et al. [13] performed the first measurements of the abdominal organs stiffness evaluating 35 healthy volunteers, the mean values being $1.59 \mathrm{~m} / \mathrm{s}$ for liver and $2.44 \mathrm{~m} / \mathrm{s}$ for spleen. In our study we obtained lower values for liver in patients without liver pathology $(1.18 \pm 0.22)$ and slightly higher spleen values $(2.60 \pm 0.42)$ for the same health group.

Bota et al. [14] used ARFI to determine ARFI spleen values in cirrhotic patients and its use in predicting the presence of portal hypertension signs (esophageal varices). The study included 57 cirrhotic patients and for a cut-off value of $2.51 \mathrm{~m} / \mathrm{s}$ (AUROC 0.91, p < 0.0001, with 85.2\% Se, 91.7\% Sp, 95.8\% PPV, 73.3\% NPV and 87.1\% accuracy). Thus confirming the hypothesis that an increase in splenic stiffness occurs with the develop- 
ment of portal hypertension. In our study, we obtained slightly higher values for spleen values in cirrhotic patients $(3.03 \pm 0.71)$.

Takuma et al. [15], Grgurevic et al. [16] and Rifai et al. [17] evaluated ARFI of the liver and the spleen of groups of healthy and cirrhotic patients. As our study, all obtained significant higher ARFI values for both organs in cirrhotic patients (for ARFI liver values the results were respectively $1.21 \mathrm{~m} / \mathrm{s}, 1.13 \pm 0.13 \mathrm{~m} / \mathrm{s}, 1.09 \pm$ $0.18 \mathrm{~m} / \mathrm{s}$ for healthy and $2.16 \mathrm{~m} / \mathrm{s}, 2.27 \pm 0.35 \mathrm{~m} / \mathrm{s}, 2.86 \pm 0.58 \mathrm{~m} / \mathrm{s}$ for cirrhotic and for ARFI spleen values the results were respectively $2.58 \mathrm{~m} / \mathrm{s}, 2.77 \pm 0.68 \mathrm{~m} / \mathrm{s}, 2.55 \pm 1.02 \mathrm{~m} / \mathrm{s}$ for healthy and $3.36 \mathrm{~m} / \mathrm{s}, 3.29 \pm 0.65 \mathrm{~m} / \mathrm{s}$, $3.25 \pm 0.56$ for cirrhotic).

Spleno-hepatic index has been studied in same literature [18] [19] and had found same contradictory results. Our results showed that, when we combine the 2 values of ARFI (hepatic and splenic) in that index, we do not find a better AUC for the diagnosis of cirrhosis.

Vermehren et al. [20] performed a study with 166 cirrhotic patients and correlate, among other hypothesis, the correlation between hepatic and splenic ARFI and the prediction of Child-Pugh B/C vs. A. They concluded that there were significant overall differences between those clinical groups. In our study, we found that there is a tendency of higher levels of liver ARFI values for higher the Child-Pugh classification (A to B and B to C), suggesting a definite trend for higher values with more severe disease.

ARFI also produced results correlated with those obtained laboratory tests: there was a statistically significant direct correlation between liver ARFI values and the laboratory results of bilirubin, ALKP, GGT, AST and AST/ALT ratio and a statistically significant direct correlation between the spleen ARFI and ALKP and AST/ALT ratio.

Furthermore, an advantage of using both techniques (B mode and ARFI) is that they are fast and convenient to perform and use a conventional US machine. Moreover, ultrasound permits the evaluation of other features such as portal vein diameter, splenomegaly and liver surface, which has been shown to be highly accurate in the diagnosis of early cirrhosis and seems to provide complementary information to that of liver stiffness.

The limitations of our study include the following: unavoidable selection bias caused by clinical diagnosis of cirrhosis without a liver biopsy; a single center study without external validation.

\section{Conclusions}

In the present study, we have found that ARFI showed excellent accuracy to determine the presence of cirrhosis. We also found that liver ARFI was better than both spleen ARFI and spleno-hepatic index for the diagnosis of cirrhosis.

We showed that there is a tendency of higher levels of liver ARFI values for higher Child-Pugh classification suggesting a definite trend for higher values with more severe disease.

We found that there is a statistically significant direct correlation between liver ARFI values and the laboratory results of bilirubin, ALKP, GGT, AST and AST/ALT ratio. In what concerns spleen ARFI values, we found a statistically significant direct correlation between the ARFI and ALKP and AST/ALT ratio.

According to the limitations mentioned, further studies are needed to overcome them, with a larger number of cirrhotic patients that will provide us with a cut-off between the groups of this classification, as a good diagnostic tool for the prediction of worse prognosis that will help in clinical practice.

\section{References}

[1] Hojun, Y. and Stephanie, R.W. (2012) New Noninvasive Ultrasound Techniques—Can They Predict Liver Cirrhosis? Ultrasound Quarterly, 28, 5-11. http://dx.doi.org/10.1097/RUQ.0b013e31824a4fc9

[2] Strader, D.B., Wright, T., Thomas, D.L. and Steeff, L.B. (2004) American Association for the Study of Liver Diseases. Diagnosis, Management and Treatment of Hepatitis C. Hepatology, 39, 1147-1171. http://dx.doi.org/10.1002/hep.20119

[3] Lok, A.S. and McMahon, B.J. (2007) Chronic Hepatitis B. Hepatology, 45, 507-539. http://dx.doi.org/10.1002/hep.21513

[4] Bedossa, P., Dargère, D. and Paradis, V. (2003) Sampling Variability of Liver Fibrosin Chronic Hepatitis C. Hepatology, 38, 1449-1457. http://dx.doi.org/10.1016/j.hep.2003.09.022

[5] Piscaglia, F., Salvatore, V., Di Donato, R., D’Onofrio, M., Gualandi, S., Gallotti, A., Peri, E., Borghi, A., Conti, F., Fattovich, G., Sagrini, E., Cucchetti, A., Andreone, P. and Bolondi, L. (2011) Accuracy of Virtual Touch Acoustic 
Radiation Force Impulse (ARFI) Imaging for the Diagnosis of Cirrhosis during Liver Ultrasonography. Ultraschall in der Medizin, 32, 167-175. http://dx.doi.org/10.1055/s-0029-1245948

[6] Friedrich-Rust, M., Nierhoff, J., Lupsor, M., Sporea, I., Fierbinteanu-Braticevici, C., Strobel, D., Takahashi, H., Yoneda, M., Suda, T., Zeuzem, S. and Herrmann, E. (2012) Performance of Acoustic Radiation Force Impulse Imaging for the Staging of Liver Fibrosis: A Pooled Meta-Analysis. Journal of Viral Hepatitis, 19, e212-e219. http://dx.doi.org/10.1111/j.1365-2893.2011.01537.x

[7] Madsen, E.L., Sathoff, H.J. and Zagzebski, J.A. (1983) Ultrasonic Shear Wave Properties of Soft Tissue and Tissue Like Material. Acoustical Society of America, 74, 1346-1355. http://dx.doi.org/10.1121/1.390158

[8] Frizzel, L.A. and Carstensen, E.L. (1976) Shear Properties of Mammalian Tissues at Low Megahertz Frequencies. Acoustical Society of America, 60, 1409-1411. http://dx.doi.org/10.1121/1.381236

[9] Lupsor, M., Badea, R., Stefanescu, H., Sparchez, Z., Branda, H., Serban, A. and Maniu, A. (2009) Performance of a New Elastographic Method (ARFI Technology) Compared to Unidimensional Transient Elastography in the Noninvasive Assessment of Chronic Hepatitis C. Preliminary Results. Journal of Gastrointestinal and Liver Diseases, 18, 303310.

[10] Zhai, L., Palmeri, M.L., Bouchard, R.R., Nightingale, R.W. and Nightingale, K.R. (2008) An Integrated Indenter-ARFI Imaging System for Tissue Stiffness Quantification. Ultrason Imaging, 30, 95-111. http://dx.doi.org/10.1177/016173460803000203

[11] Nightingale, K., Soo, M.S., Nightingale, R. and Trahey, G. (2002) Acoustic Radiation Force Impulse Imaging: In Vivo Demonstration of Clinical Feasibility. Ultrasound in Medicine and Biology, 28, 227-235. http://dx.doi.org/10.1016/S0301-5629(01)00499-9

[12] Mauldin Jr., F.W., Zhu, H.T., Behler, R.H., Nichols, T.C. and Gallippi, C.M. (2008) Robust Principal Component Analysis and Clustering Methods for Automated Classification of Tissue Response to ARFI Excitation. Ultrasound in Medicine and Biology, 34, 309-325. http://dx.doi.org/10.1016/j.ultrasmedbio.2007.07.019

[13] Gallotti, A., D’Onofrio, M. and Pozzi Mucelli, R. (2010) Acoustic Radiation Force Impulse (ARFI) Technique in Ultrasound with Virtual Touch Tissue Quantification of the Upper Abdomen. La Radiologia Medica, 115, 889-897. http://dx.doi.org/10.1007/s11547-010-0504-5

[14] Bota, S., Sporea, I., Sirli, R., Popescu, A., Danila, M., Sendroiu, M. and Focsa, M. (2010) Spleen Assessment by Acustic Radiation Force Impulse Elastography (ARFI) for Prediction of Liver Cirrhosis and Portal Hypertension. Medical Ultrasonography, 12, 213-217.

[15] Takuma, Y., Nouso, K., Morimoto, Y., Tomokuni, J., Sahara, A., Toshikuni, N., Takabatake, Y., Shimomura, H., Doi, A., Sakakibara, I., Matsueda, K. and Yamamoto, H. (2013) Measurement of Spleen Stiffness by Acoustic Radiation Force Impulse Imaging Identifies Cirrhotic Patients with Esophageal Varices. Gastroenterology, 144, 92-101. http://dx.doi.org/10.1053/j.gastro.2012.09.049

[16] Grgurevic, I., Cikara, J., Horvat, I., Lukic, K., Heinzl, R., Banic, M., Kujundzic, M. and Brkljacic, B. (2011) Noninvasive Assessment of Liver Fibrosis with Acoustic Radiation Force Impulse Imaging: Increased Liver and Splenic Stiffness in Patients with Liver Fibrosis and Cirrhosis. Ultraschall in der Medizin, 32, 160-166. http://dx.doi.org/10.1055/s-0029-1245807

[17] Rifai, K., Cornberg, J., Bahr, M., Mederacke, I., Potthoff, A., Wedemeyer, H., Manns, M. and Gebel, M. (2011) ARFI Elastography of the Spleen Is Inferior to Liver Elastography for the Detection of Portal Hypertension. Ultraschall in der Medizin, 32, E24-E30. http://dx.doi.org/10.1055/s-0031-1281771

[18] Berzigotti, A., Abraldes, J.G., Tandon, P., Erice, E., Gilabert, R., Garcia-Pagan, J.C. and Bosch, J. (2010) Ultrasonographic Evaluation of Liver Surface and Transient Elastography in Clinically Doubtful Cirrhosis. Journal of Hepatology, 52, 846-853. http://dx.doi.org/10.1016/j.jhep.2009.12.031

[19] Sporea, I., Bota, S., Sirli, R., Danila, M. and Sendroiu, M. (2011) Splenohepatic Index in Acoustic Radiation Force Impulse Elastography (ARFI) Can Increase the Accuracy for the Portal Hypertension Prediction in Cirrhotic Patients? Journal of Hepatology, 54, S81-S82. http://dx.doi.org/10.1016/S0168-8278(11)60193-1

[20] Vermehren, J., Polta, A., Zimmermann, O., Herrmann, E., Poynard, T., Hofmann, W.P., Bojunga, J., Sarrazin, C., Zeuzem, S. and Friedrich-Rust, M. (2012) Comparison of Acoustic Radiation Force Impulse Imaging with Transient Elastography for the Detection of Complications in Patients with Cirrhosis. Liver International, 32, 852-858. http://dx.doi.org/10.1111/j.1478-3231.2011.02736.x 


\section{Abbreviations}

ALKP_Alkaline Phosphatase

ALT_-Alanine Aminotransferase

ARFI-Acoustic Radiation Force Impulse

AST-Aspartate Aminotransferase

AUC-Area under Curve

Bilirubin-Total Bilirubin

GGT- $\gamma$-Glutamyl Transpeptidase

HBV-Hepatitis B Virus

HCV-Hepatitis C Virus

PBC—Primary Biliary Cirrhosis

Se-Sensitivity

Sp-Specificity 